\title{
On strong shift equivalence over a Boolean semiring
}

\author{
KI HANG KIM AND FRED W. ROUSH \\ Mathematics Research Group, Alabama State University, \\ Montgomery, Alabama 36195, USA
}

(Received 29 October 1984 and revised 1 July 1985)

\begin{abstract}
Shift equivalence is the relation between $A, B$ that there exists $S, R$, $n>0$ with $R A=B R, A S=S B, S R=A^{n}, R S=B^{n}$. Strong shift equivalence is the equivalence relation generated by these equations with $n=1$. We prove that for many Boolean matrices strong shift equivalence is characterized by shift equivalence and a trace condition. However, we also show that if $\boldsymbol{A}$ is strongly shift equivalent to $B$, then there exists a homomorphism from an iterated directed edge graph of $A$ to the graph of $B$ preserving the traces of powers. This yields results on colourings of iterated directed edge graphs and might distinguish new strong equivalence classes.
\end{abstract}

\section{Introduction}

Two matrices $A, B$ over a semiring are said to be shift equivalent if there exist matrices $R, S$ with $R A=B R, A S=S B, S R=A^{n}, R S=B^{n}, n>0$. They are said to be strongly shift equivalent if there exists a sequence $A_{i}=S_{i} R_{i}, A_{i+1}=R_{i} S_{i}, A=A_{0}$, $B=A_{n}$ (For $n=1$, the equations are equivalent to $A=S R$ and $B=R S$.) It is important that $R$ and $S$ need not be square. The relations thus defined, shift equivalence and strong shift equivalence, will be abbreviated s.e. and s.s.e.

We shall be concerned with two specific semirings: the semiring $\mathbb{Z}^{+}$of non-negative integers and the Boolean semiring $\mathfrak{B}=\{0,1\}$ with the usual multiplication and with addition being 'logical or' where $1+1=1$. A semiring homomorphism $\mathbb{Z}^{+} \rightarrow \mathfrak{B}$ is obtained by associating with each matrix $A$ over $\mathbb{Z}^{+}$its 'Boolean image' where all positive integer entries have been replaced by 1 . Observe that this homomorphism is compatible with matrix products. Therefore, s.s.e. of Boolean images is a necessary condition for s.s.e. of matrices over $\mathbb{Z}^{+}$.

S.e. over $\mathbb{Z}^{+}$is used in the study [8] and [9] of subshifts of finite type and from them of diffeomorphisms. For an $n$-square $(0,1)$-matrix $A$, let $X$ be the set of all sequences $\left(x_{i}\right), i \in \mathbb{Z}, x_{i} \in \mathbf{n}$ where $\mathbf{n}=\{1, \ldots, n\}$ such that the $\left(x_{i}, x_{i+1}\right)$-entry of $A$ is always 1 . The shift map is the mapping $\widetilde{S}: X \rightarrow X$ sending $\left(x_{i}\right)$ to $\left(y_{i}\right)$ and $y_{i}=x_{i+1}$. Let $X$ be a compact metrizable space. The pair $(X, \Im)$ is called a subshift of finite type. Subshifts $(X, \Im)$ and $\left(X^{\prime}, \widetilde{S}^{\prime}\right)$ are said to be topologically conjugate if there is a homeomorphism $f$ for $X$ and $X^{\prime}$ such that $\varsigma^{\prime} f=f \subseteq$.

Williams [8], [9] proved that topological conjugacy of two subshifts is equivalent to strong shift equivalence over $\mathbb{Z}^{+}$of their defining matrices. 
It is an open question whether s.e. coincides with s.s.e. over $\mathbb{Z}^{+}$. If they do coincide then s.e. would completely decide topological conjugacy of subshifts. S.e. is the more easily computed. In fact, in [5] a decision procedure for s.e. was found in the case that all eigenvalues are distinct. Additional results are in [1], [7].

S.s.e. is more complicated. Here as a first step we show that over $\mathfrak{B}$ a large class of matrices has the property that two matrices in it are strong shift equivalent if and only if they are shift equivalent and the (Boolean) traces of every power of corresponding indecomposable components are equal. However, there are many Boolean matrices not covered by this result, especially those of trace zero.

Example. Over $\mathbb{Z}^{+}$the matrices

with

$$
A=\left[\begin{array}{lll}
0 & 3 & 1 \\
1 & 0 & 1 \\
1 & 0 & 0
\end{array}\right], \quad B=\left[\begin{array}{lll}
0 & 2 & 1 \\
1 & 0 & 1 \\
1 & 1 & 0
\end{array}\right]
$$

$$
R=\left[\begin{array}{lll}
1 & 2 & 2 \\
1 & 1 & 1 \\
1 & 1 & 0
\end{array}\right], \quad S=\left[\begin{array}{rrr}
7 & 42 & 8 \\
17 & 6 & 17 \\
16 & 9 & 15
\end{array}\right], \quad n=6
$$

are shift equivalent. Their Boolean images are strong shift equivalent (theorem 7.1 of this paper) but non-trivially.

First we show how to express all s.e. over $\mathfrak{B}$ as compositions of elementary ones using operations on rows and columns. Then we show s.s.e. gives an s.s.e. on each indecomposable component (main diagonal block in a block triangular form). Then we show the indecomposable case can be reduced to the primitive case (for some $n, A^{n}>0$ ). In the primitive case, we first duplicate rows and columns to obtain a Hamiltonian circuit then split the columns along it in turn and add ones. This gives the matrix of all ones. We extend this back to the indecomposable and general cases. We show $\operatorname{Tr}(A)$ and thus $\operatorname{Tr}\left(A^{n}\right)$ is a strong shift invariant. Here $\operatorname{Tr}(A)$ denotes the trace of $A$.

In the case of trace zero, we give a necessary condition of a homomorphism of graphs obtained from our elementary operations.

\section{Results from Boolean matrix theory}

For brevity, let $J$ denote a Boolean matrix all of whose entries are 1.

Definition. A Boolean matrix $A$ is primitive (aperiodic) if $A^{n}=J$ for some $n>0$. It is indecomposable (irreducible) if

$$
\sum_{n=1}^{\infty} A^{n}=J
$$

Results on Boölean matrices can be found in [4], [6].

THEOREM 2.1. [4, p. 185] The following are equivalent for an $n$-square Boolean matrix $A$ :

(1) $A$ is indecomposable;

(2) $\sum_{r=1}^{n-1} A^{r}=J$ 
(3) there does not exist a permutation matrix $P$ with $P A P^{T}$ having the block form with main diagonal blocks square.

$$
\left[\begin{array}{ll}
B & 0 \\
C & D
\end{array}\right]
$$

Definition. The directed graph (d.g.) associated with an $n$-square Boolean matrix $A$ has vertices $v_{1}, \ldots, v_{n}$ and a directed edge from $v_{i}$ to $v_{j}$ if and only if $a_{i j}=1$. A d.g. is said to be strongly connected if for all $v_{i}, v_{j}$ there exists a sequence of vertices $v_{i}=x_{1}, x_{2}, \ldots, x_{r+1}=v_{j}$ such that for $i<r+1, x_{i}$ is connected to $x_{i+1}$ by a directed edge.

A matrix is indecomposable if and only if its graph is strongly connected. The following result is equivalent to finding the strong components of a d.g.

In block forms, we assume main diagonal blocks are square whenever the setting is such that the matrix must be square.

TheORem 2.2. [4, p. 222] For any Boolean matrix $A$ there exists a permutation matrix $P$ such that PAP $P^{T}$ has a block triangular form

$$
\left[\begin{array}{cccc}
A_{11} & 0 & \cdots & 0 \\
A_{21} & A_{22} & \cdots & 0 \\
\cdots & \cdots & \cdots & \cdots \\
A_{n 1} & A_{n 2} & \cdots & A_{n n}
\end{array}\right]
$$

where all main diagonal blocks are indecomposable or zero.

Definition. For a Boolean matrix $A$, let $k$ be the least positive integer such that $A^{k+d}=A^{k}$ for some $d>0$. Then $k$ is the index and the least $d>0$ is the period. If $k=1$ then $A$ is periodic. The period is the 'eventual' period.

THEOREM 2.3. [4, p. 188] An indecomposable Boolean matrix of period $d$ has the form, for some permutation matrix $P$

$$
A=P\left[\begin{array}{ccccc}
0 & A_{12} & 0 & \cdots & 0 \\
0 & 0 & A_{23} & \cdots & 0 \\
\ldots & \cdots & \cdots & \cdots & \ldots \\
A_{d 1} & 0 & 0 & \cdots & 0
\end{array}\right] P^{T}
$$

If it is periodic, all blocks $A_{i, i+1}, A_{d 1}$ can be taken as $J$.

Definition. A Boolean matrix $A$ is difunctional if each two rows are equal or have zero inner product. It is regular if $\boldsymbol{A X A}=\boldsymbol{A}$ for some $X$. Every difunctional matrix is regular where $X=A^{T}$.

An $n$-square Boolean matrix $A$ is called non-singular if it is regular and has row rank $n$, that is, no rows are linear Boolean combinations of other rows. It is reflexive (irreflexive) if $a_{i i}=1\left(a_{i i}=0\right)$ for all $i$. (See [4] and [6].) Every non-singular matrix $A$ has a unique Thierrin-Vagner inverse $X$ (i.e. $A X A=A$ and $X A X=X$ ). There exists a unique permutation matrix $P \leq A$ for non-singular $A$.

Every Boolean matrix $A$ can be factored as $B C$ ( $B, C$ rectangular) where $B$ has at most one 1 per column and $C$ has exactly one 1 per row. Take one row of $C$ for 
each 1 entry of $A$, containing only that entry. Let $b_{i j}=1$ only for those $j$ corresponding to entries of $A$ in row $i$. If we reverse the product to take $C B$, we get a matrix often used below, the matrix of the directed edge graph $G_{E}$ of $A$. Vertices of $G_{E}$ are in 1-1 correspondence with directed edges of $G$ in the directed graph of $A$ and edges with joined pairs of edges. This is different from the undirected edge graph.

\section{Reduction of s.s.e.}

We need the following propositions.

Proposition 3.1. S.s.e. can be broken down into s.s.e. in which $R$ is a permutation matrix or has the form

$$
\left[\begin{array}{cc}
I & 0 \\
F & 0
\end{array}\right], \text { or }\left[\begin{array}{cc}
I & F^{T} \\
0 & 0
\end{array}\right]
$$

where $F$ has at most one 1 per row.

Proof. We can factor any matrix $R$ as a product of matrices with at most one 1 in each row (column). By introducing permutations we may change to the above form. Factoring $R$ as $R_{1} R_{2}$ factors the s.e.

$$
R_{1} R_{2} S=B \sim R_{2} S R_{1} \sim S R_{1} R_{2}=A .
$$

We introduce a standard s.s.e. as follows: write $A=R S$ where we number the ones of $B$ as $1, \ldots, k$, and $r_{i j}=1$ if and only if $j \leq k$ and the $j$ th one of $B$ lies in row $i$, and $s_{j m}=1$ if and only if $j \leq k$ and the $j$ th one of $B$ lies in column $m$. Then $R(S)$ has at most one 1 per row (column). Then $S R$ is difunctional and is therefore regular.

Proposition 3.2. Every Boolean matrix is strongly shift equivalent to a regular Boolean, in fact one satisfying $A A^{T} A=A$.

Proof. Difunctional Boolean matrices have this property.

Proposition 3.3. Suppose a Boolean matrix $M$ is periodic having the form with $A$ non-singular

$$
\left[\begin{array}{cc}
A & A C \\
B A & B A C
\end{array}\right]
$$

i.e. the first $r$ rows and columns form a basis. Then $M$ is strongly shift equivalent to $A$.

Proof. Clearly

$$
\left[\begin{array}{l}
I \\
B
\end{array}\right]\left[\begin{array}{ll}
A & A C
\end{array}\right]=M, \quad\left[\begin{array}{ll}
A & A C
\end{array}\right]\left[\begin{array}{c}
I \\
B
\end{array}\right]=A+A C B .
$$

Since $A$ is non-singular, there exists a permutation matrix $P \leq A$. Let $P^{n}=I$. But periodicity implies that $A^{d n+1} \leq A$ and $A^{d n+1} \geq P^{n d} A=A$. Also by periodicity $A C B A A^{d n-1} \leq A^{d n+1}=A$. So $A C B I \leq A C B A^{d n} \leq A$.

Proofs of the next two propositions are in [5].

Proposition 3.4. Two periodic non-singular Boolean matrices are shift equivalent if and only if they are conjugate by a permutation matrix. 
Proposition 3.5. Shift equivalence over $\mathfrak{B}$ is decided as follows. Every matrix $A$ is shift equivalent to a periodic power of itself. Two periodic matrices are shift equivalent if and only if after deletion of dependent rows and columns, they are conjugate by a permutation matrix.

Next we mention certain row and column operations which are always s.s.e. Here $A_{i^{*}}\left(A_{*_{i}}\right)$ denotes the $i$ th row (column) of $A$.

Proposition 3.6. We can replace $A_{*_{i}}$ by two columns whose sum is $A_{*_{i}}$ and then take two copies of $A_{i^{*}}$.

Proof. Let $S$ be $A$ after $A_{*_{i}}$ is split into two columns and $R$ be the matrix which adds those two columns and preserves all others.

Proposition 3.7. If $A_{*_{i}} \leq A_{*_{j}}$, we may delete any set of elements in $A_{*_{j}}$ which are 1 in $A_{*_{i}}$ and then add $A_{*_{j}}$ to $A_{i *}$.

Proof. Let $S$ be the matrix obtained by possibly changing elements in $A_{* j}$ which are 1 in $A_{*_{i}}$ to zero. Let $R$ be the matrix such that $r_{i j}=1$ and otherwise $R$ equals the identity. Then $S R=A$ and $R S$ is the matrix considered.

Proposition 3.8. Suppose $A_{i^{*}}$ is dependent and is the sum of $A_{k^{*}}$ for $k \in K$, for some set $K$, $i \notin K$. Then we may delete $A_{i *}$, add $A_{*_{i}}$ to $A_{*_{k}}$ for $k \in K$, and delete $A_{*_{i} \text {. }}$

Proof. Let $S$ be the matrix which is an $(n-1)$-square identity with a row inserted in place $i$ having ones in locations $K$. Let $R$ be $A$ with row $i$ deleted. Then $S R=A$, and $R S$ is the matrix considered.

Example. It may not be possible to delete the row and columns of a zero main diagonal block. Consider

$$
\left[\begin{array}{llll}
1 & 0 & 0 & 0 \\
1 & 0 & 0 & 0 \\
0 & 1 & 0 & 0 \\
0 & 0 & 1 & 1
\end{array}\right] .
$$

This is s.e. to its power

$$
\left[\begin{array}{llll}
1 & 0 & 0 & 0 \\
1 & 0 & 0 & 0 \\
1 & 1 & 0 & 0 \\
1 & 1 & 1 & 1
\end{array}\right]
$$

and so to

$$
\left[\begin{array}{ll}
1 & 0 \\
1 & 1
\end{array}\right]
$$

not to

$$
\left[\begin{array}{ll}
1 & 0 \\
0 & 1
\end{array}\right]
$$

Let $a_{i j}^{(n)}$ denote the $(i, j)$-entry of $A^{n}$. 
Theorem 3.10. Any s.s.e. between $A$ and $B$ gives an s.s.e. between the irreducible main diagonal blocks of $A, B$.

Proof. We put $A, B$ into block triangular form. Let $R, S$ give an s.s.e. Suppose that $r_{i m} s_{m j}=r_{c d} s_{d e}=1$ for $b_{i j}, b_{c e}$ in the same non-zero main diagonal block of $B$. Then $s_{m j} b_{j c}^{(u)} r_{c d}$ is non-zero in $A^{u+1}$ for some $u$ since the $(j, c)$-block is indecomposable. So some entry $a_{m d}^{(u+1)}=1$. Likewise some entry $a_{d m}^{(v+1)}=1$. This by the block form of all powers of $A$ means $d, m$ lie in the same block of $A$. So this block of $B$ is the product of a single pair of blocks from $R, S$. If we take their product in reverse order we get a single main diagonal block of $A$, by symmetry.

Corollary 3.11. If $A, B$ are irreducible (over $\mathfrak{B}$ or $\mathbb{Z}^{+}$) of period $d$ and are strong shift equivalent, we may take every intermediate matrix in the strong shift equivalence to be irreducible of period $d$.

Proof. We can take a single main diagonal block at each step. Since the period is an s.e. invariant (the period of $\boldsymbol{A}$ equals that of a high power which is periodic) by proposition 3.7 it is an invariant.

M. Boyle has observed that the next result considered over $\mathbb{Z}^{+}$has a topological interpretation [2]. If all words must have letters $x_{i}$ in a subset $X_{i+k}$ modulo $d$, for some $k$, then map $\left(\cdots x_{k} x_{k+1} \cdots x_{k+d} x_{k+d+1} \cdots\right)$ to $\left(\cdots x_{k} x_{k} \cdots x_{k+d} x_{k+d} \cdots\right)$. This gives a topological mapping corresponding to the matrix equation. If we arrange things so all matrix entries are 0 and 1 it is a conjugacy.

THEOREM 3.12. An indecomposable matrix of period $d$ can be put into the form

$$
\left[\begin{array}{ccccc}
0 & A_{1} & 0 & \cdots & 0 \\
0 & 0 & A_{2} & \cdots & 0 \\
\cdots & \cdots & \cdots & \cdots & \cdots \\
0 & 0 & 0 & \cdots & A_{d-1} \\
A_{d} & 0 & 0 & \cdots & 0
\end{array}\right]
$$

by conjugating it with a permutation. It is strong shift equivalent to

$$
\left[\begin{array}{ccccc}
0 & I & 0 & \cdots & 0 \\
0 & 0 & I & \cdots & 0 \\
\cdots & \cdots & \cdots & \cdots & \cdots \\
b & 0 & 0 & \cdots & 0
\end{array}\right], \quad b=A_{1} \cdots A_{d}
$$

Two matrices of this form are strong shift equivalent if their lower left-hand blocks are. Proof. The first statement is theorem 2.3 found in Kim [4]. For the second, as an intermediate step we use the matrices $M\langle k\rangle$ having the form above with $M_{i, i+1}\langle k\rangle=I$ for $i<k$ and $M_{k, k+1}\langle k\rangle=A_{1} \cdots A_{k}$ and $M_{i, i+1}\langle k\rangle=A_{i}$ for $i>k$ (and $M_{n 1}\langle k\rangle$ has this form).

Let $R\langle k\rangle$ have all blocks zero except $R_{i, i+1}\langle k\rangle=I$ for $i\left\langle k\right.$ and $R_{i, i+1}\langle k\rangle=A_{i}$ for $i \geq k$ (and $R_{n}\langle k\rangle$ ). Let $S\langle k\rangle$ have all blocks zero except $S_{k k}\langle k\rangle=A_{1} \cdots A_{k-1}$ and $S_{i i}\langle k\rangle=I$ for $i \neq k$. Then $S\langle k\rangle R\langle k\rangle=M\langle k\rangle$ and $R\langle k\rangle S\langle k\rangle=M\langle k-1\rangle$. This proves the second statement. Suppose $A_{n 1}=X Y, B_{n 1}=Y X$. Let $R_{n 1}=X, R_{i, i+1}=I$ and other 
blocks be zero. Let $S\langle k\rangle$ be a block identity matrix with the $(k, k)$-block replaced by $Y$. Then

$$
S\langle n\rangle R=\left[\begin{array}{ccccc}
0 & I & 0 & \ldots & 0 \\
0 & 0 & I & \cdots & 0 \\
\cdots & \ldots & \ldots & \ldots & \ldots \\
Y X & 0 & 0 & \ldots & 0
\end{array}\right], \quad R S\langle 1\rangle=\left[\begin{array}{ccccc}
0 & I & 0 & \cdots & 0 \\
0 & 0 & I & \cdots & 0 \\
\ldots & \ldots & \ldots & \cdots & \ldots \\
X Y & 0 & 0 & \cdots & 0
\end{array}\right],
$$

and $R S\langle k\rangle=S\langle k-1\rangle R$. This proves the last statement.

\section{Matrices with positive trace}

We begin with the following proposition.

Proposition 4.1. The process of splitting column $j$ in two and adding an identical copy of row $i$ preserves the property of indecomposability of a Boolean matrix if both new columns are non-zero.

Proof. The graph of the larger matrix is obtained by splitting vertex $j$ and letting all arrows go out of both copies but dividing the arrows in. Let $j_{1}, j_{2}$ be the new vertices and $k$ another vertex, and $m$ a vertex with an arrow into $j_{1}$. Then there exists a path from $j_{1}$ to $k$. There exists a path from $k$ to $m$ (if $j$ occurred it could always be replaced by $j_{1}$ or $j_{2}$ ). And we can go from $k$ to $m$ to $j_{1}$. This says the graph of the new matrix is strongly connected, since there exists a path from any vertex to any other.

Proposition 4.2. Every indecomposable Boolean matrix $A$ with a row $(1,1, \ldots, 1)$ is strong shift equivalent to $J$.

Proof. Suppose we have a set $S$ of rows having all ones. By indecomposability $a_{i j}=1$ for some $i \notin S, j \in S$ unless $A=J$. Since $j \in S$ and every row $A_{m^{*}} \leq A_{j^{*}}$ we can add column $j$ to column $m$. This will add column $j$ to every column. Thus $A_{i *}$ is now $(1,1, \ldots, 1)$. Continue to obtain $J$.

The next example illustrates how we can deal with an indecomposable matrix of positive trace.

Example. Let

$$
A=\left[\begin{array}{llll}
1 & 1 & 0 & 0 \\
0 & 1 & 1 & 0 \\
0 & 0 & 1 & 1 \\
1 & 0 & 0 & 1
\end{array}\right]
$$

Split columns 2, 3 in two and duplicate rows 2,3 in turn, to obtain

$$
\left[\begin{array}{llllll}
1 & 1 & 1 & 0 & 0 & 0 \\
0 & 0 & 1 & 1 & 1 & 0 \\
0 & 0 & 1 & 0 & 1 & 0 \\
0 & 0 & 0 & 0 & 1 & 1 \\
0 & 0 & 0 & 0 & 1 & 1 \\
1 & 0 & 0 & 0 & 0 & 1
\end{array}\right] .
$$


Now $A_{*_{2}} \leq A_{*_{1}}$ so add $A_{1^{*}}$ to $A_{2^{*}}$, giving

$$
\left[\begin{array}{llllll}
1 & 1 & 1 & 0 & 0 & 0 \\
1 & 1 & 1 & 1 & 1 & 0 \\
0 & 0 & 1 & 0 & 1 & 0 \\
0 & 0 & 0 & 0 & 1 & 1 \\
0 & 0 & 0 & 0 & 1 & 1 \\
1 & 0 & 0 & 0 & 0 & 1
\end{array}\right] .
$$

Since $A_{*_{4}} \leq A_{*_{2}}$ we can add $A_{2^{*}}$ to $A_{4^{*}}$ :

$$
\left[\begin{array}{llllll}
1 & 1 & 1 & 0 & 0 & 0 \\
1 & 1 & 1 & 1 & 1 & 0 \\
0 & 0 & 1 & 0 & 1 & 0 \\
1 & 1 & 1 & 1 & 1 & 1 \\
0 & 0 & 0 & 0 & 1 & 1 \\
1 & 0 & 0 & 0 & 0 & 1
\end{array}\right] .
$$

Now we have a row of all 1 so we can obtain $J$.

Proposition 4.3. Every indecomposable Boolean matrix with positive trace is strong shift equivalent to $J$.

Proof. Let $A$ be indecomposable with $a_{i i}=1$. We can find a sequence $i=i_{1}, \ldots, i_{k}=i$ such that $a_{i_{i} i_{r+1}}=1$ for all $r$ and the $i_{j}$ include every vertex by indecomposability. For instance for every $n$ take a path from $n$ to $n+1$ of this nature.

Duplicate rows and columns so that the matrix has as many copies of row and column $i$ as the sequence does, rearranging them in the order of the sequence. So $a_{11}=1, a_{12}=a_{23}=\cdots=a_{k-1, k}=1$.

Now we in turn split each column $j=2,3, \ldots, k-1$ in two columns $j_{1}, j_{2}$, the first having a 1 only in location $i_{1}$ where $i=j-1$ and the second equal to column $j$. Duplicate row $j$.

Now we note that $A_{*_{2}} \leq A_{*_{1}}$ so add $\boldsymbol{A}_{1^{*}}$ to $\boldsymbol{A}_{2_{1}}{ }^{*}$. Inductively we assume by this process that if $i=j-1, A_{i_{1}}$ has ones in all locations prior to and including $j_{1}, j_{2}$.

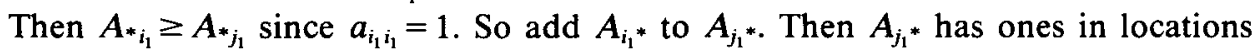
prior to and including $(j+1)_{1},(j+1)_{2}$. Then $A_{*_{i_{1}}} \geq A_{*_{j_{1}}}$ so we obtain a row with all ones. Now we apply proposition 4.2 .

\section{Matrices with a general trace condition}

We are ready for general characterization theorems.

THEOREM 5.1. Let $A, B$ be the square Boolean matrices. If $A$ is strong shift equivalent to $B$ and $A_{i}, B_{i}$ are the indecomposable components of $A, B$ then with some labelling such that $A_{i}$ is shift equivalent to $B_{i}$ we have $\operatorname{Tr}\left(A_{i}^{n}\right)=\operatorname{Tr}\left(B_{i}^{n}\right)$ for all $n>0$. Here $\operatorname{Tr}(A)$ denotes the trace of $A$.

Proof. An s.s.e. between $A, B$ gives one between their indecomposable components, 
and between $A^{n}$ and $B^{n}$ since

$$
A^{n}=(S R)^{n}=S R(S R)^{n-1}, \quad B^{n}=R(S R)^{n-1} S .
$$

And

$$
\operatorname{Tr}(A B)=\sum a_{i j} b_{j i}=\sum b_{j i} a_{i j}=\operatorname{Tr}(B A)
$$

so

$$
\operatorname{Tr}(R S)=\operatorname{Tr}(B)=\operatorname{Tr}(S R)=\operatorname{Tr}(A) .
$$

Corollary 5.2. S.s.e. does not coincide with s.e. for Boolean matrices.

Remark. However, this does not affect the $\mathbb{Z}^{+}$or $\mathbb{Q}^{+}$cases since $\operatorname{Tr}\left(A^{n}\right)$ and $\operatorname{Tr}\left(B^{n}\right)$ will always be equal.

THEOREM 5.3. Let $A$ be an indecomposable Boolean matrix of period d. If $\operatorname{Tr}\left(A^{d}\right) \neq 0$ then $A$ is strong shift equivalent to

$$
\left[\begin{array}{ccccc}
0 & J & 0 & \cdots & 0 \\
0 & 0 & J & \cdots & 0 \\
\cdots & \cdots & \cdots & \cdots & \cdots \\
J & 0 & 0 & \cdots & 0
\end{array}\right]
$$

Proof. By theorems 2.3 and 5.3 we may assume $A$ has the form

$$
\left[\begin{array}{ccccc}
0 & I & 0 & \cdots & 0 \\
0 & 0 & I & \cdots & 0 \\
\cdots & \cdots & \cdots & \cdots & \cdots \\
X & 0 & 0 & \cdots & 0
\end{array}\right],
$$

where $X=A_{12} \cdots A_{d 1}$ is primitive and has a main diagonal 1 since $A^{d}$ has as its main diagonal blocks $X$ and matrices strong shift equivalent to it. If $X$ were decomposable these blocks would give a place where $\sum_{m=1}^{\infty} A^{m}$ is zero.

Now we can take an s.s.e. on $X$ to reduce it to $J$. Finally, we have

$$
\left[\begin{array}{ccccc}
0 & I & 0 & \cdots & 0 \\
0 & 0 & I & \cdots & 0 \\
\cdots & \cdots & \cdots & \cdots & \cdots \\
J & 0 & 0 & \cdots & 0
\end{array}\right] .
$$

We can obtain $J$ in the other non-zero blocks by factoring out and remultiplying matrices of the form

$$
\left[\begin{array}{ccccc}
I & 0 & 0 & \cdots & 0 \\
0 & I & 0 & \cdots & 0 \\
\cdots & \cdots & \cdots & \cdots & \cdots \\
0 & 0 & J & \cdots & 0 \\
\cdots & \cdots & \cdots & \cdots & \cdots \\
0 & 0 & 0 & \cdots & I
\end{array}\right]
$$

This proves the theorem. 
THeOREM 5.4. Let $A$ be a Boolean matrix such that every non-zero indecomposable component $A_{i}$ of period $d_{i}$ satisfies $\operatorname{Tr}\left(A_{i}^{d_{i}}\right) \neq 0$. Then $A$ is strong shift equivalent to a periodic non-singular matrix.

Proof. First we eliminate zero main diagonal blocks using

$$
\left[\begin{array}{ccc}
A & 0 & 0 \\
B & 0 & 0 \\
C & E & F
\end{array}\right]=\left[\begin{array}{cc}
A & 0 \\
B & 0 \\
C & I
\end{array}\right]\left[\begin{array}{ccc}
I & 0 & 0 \\
0 & E & F
\end{array}\right] .
$$

This does not change non-zero main diagonal blocks. Next we take s.e. on the main diagonal blocks to make them periodic.

We can extend any s.s.e. on a main diagonal block to the entire matrix. Let $U V=A_{r r}$

$$
\left[\begin{array}{ccccccc}
A_{11} & \cdots & 0 & \cdots & 0 & \cdots & 0 \\
\cdots & \cdots & \cdots & \cdots & \cdots & \cdots & \cdots \\
A_{r 1} & \cdots & U & \cdots & 0 & \cdots & 0 \\
\cdots & \cdots & \cdots & \cdots & \cdots & \cdots & \cdots \\
A_{k 1} & \cdots & 0 & \cdots & I & \cdots & 0 \\
\cdots & \cdots & \cdots & \cdots & \cdots & \cdots & \cdots \\
A_{m 1} & \cdots & 0 & \cdots & 0 & \cdots & I
\end{array}\right] \times\left[\begin{array}{ccccccc}
I & \cdots & 0 & \cdots & 0 & \cdots & 0 \\
\cdots & \cdots & \cdots & \cdots & \cdots & \cdots & \cdots \\
0 & \cdots & V & \cdots & 0 & \cdots & 0 \\
\cdots & \cdots & \cdots & \cdots & \cdots & \cdots & \cdots \\
0 & \cdots & A_{k r} & \cdots & A_{k k} & \cdots & 0 \\
\cdots & \cdots & \cdots & \cdots & \cdots & \cdots & \cdots \\
0 & \cdots & A_{m r} & \cdots & A_{m k} & \cdots & A_{m m}
\end{array}\right]
$$

That is, in the first factor all blocks $B_{i j}$ for $i, j>r$ are taken 0 except the main diagonal blocks which are $I$. Then if we rearrange the factors, the $r$ th main diagonal block has $V U$ for $U V$ and the other diagonal blocks have not changed. Therefore, we can take the block $A_{r r}$ to have the desired form

$$
\left[\begin{array}{ccccc}
0 & J & 0 & \cdots & 0 \\
0 & 0 & J & \cdots & 0 \\
\cdots & \cdots & \cdots & \cdots & \cdots \\
J & 0 & 0 & \cdots & 0
\end{array}\right] .
$$

We introduce a sub-blocking of the entire matrix according to the blocking just given on the main diagonal blocks. We next make all these sub-blocks consist entirely of zeros or entirely of ones. To do this, we use the same s.e. as the last one, but we let $V=A_{r}, U=I$. We start at the lower right and go up. The row blocking on rows below $r$ is preserved, since the $A_{i j}$ have this blocking for $i>r$ and they are left-hand factors of the result. $A_{r^{*}}$ has also the blocking since $A_{r r}$ is a left-hand factor. This makes the rows correctly blocked. Then use the s.e. with $V=I, U=A_{r r}$ Columns after $r$ are unchanged and column $r$ has $A_{r r}$ as a right-hand factor, so is correctly blocked. The blocking on rows below $r$ is preserved since the $A_{k r}$ are left-hand factors. Rows above or equal to $r$ are unchanged. This makes all rows and columns correctly blocked.

Next we may replace all the blocks within blocks by single entries since they are identically 1 or 0 . This means the main diagonal blocks are permutation matrices: 


$$
\left[\begin{array}{ccccc}
0 & 1 & 0 & \cdots & 0 \\
0 & 0 & 1 & \cdots & 0 \\
\cdots & \ldots & \ldots & \ldots & \ldots \\
1 & 0 & 0 & \ldots & 0
\end{array}\right]
$$

(Take an s.s.e. where $R, S$ are block identity matrices.)

Now we show that unless the matrix is periodic, we can increase the number of 1 entries. We factor $A$ as, where $E_{m k}=A_{m m}^{-1} A_{m k}$,

$$
\left[\begin{array}{ccccccc}
A_{11} & \cdots & 0 & \cdots & 0 & \cdots & 0 \\
\cdots & \cdots & \cdots & \cdots & \cdots & \cdots & \cdots \\
A_{r 1} & \cdots & A_{r r} & \cdots & 0 & \cdots & 0 \\
\cdots & \cdots & \cdots & \cdots & \cdots & \cdots & \cdots \\
A_{k 1} & \cdots & A_{k r} & \cdots & A_{k k} & \cdots & 0 \\
\cdots & \cdots & \cdots & \cdots & \cdots & \cdots & \cdots \\
A_{m 1} & \cdots & A_{m r} & \cdots & 0 & \cdots & A_{m m}
\end{array}\right] \times\left[\begin{array}{ccccccc}
I & \cdots & 0 & \cdots & 0 & \cdots & 0 \\
\cdots & \cdots & \cdots & \cdots & \cdots & \cdots & \cdots \\
0 & \cdots & I & \cdots & 0 & \cdots & 0 \\
\cdots & \cdots & \cdots & \cdots & \cdots & \cdots & \cdots \\
0 & \cdots & E_{k r} & \cdots & I & \cdots & 0 \\
\cdots & \cdots & \cdots & \cdots & \cdots & \cdots & \cdots \\
0 & \cdots & 0 & \cdots & E_{m k} & \cdots & I
\end{array}\right] .
$$

That is, in the first factor we take all off main diagonal blocks $A_{i j} i, j \geq k$ as 0 . In the second factor we put an $E_{i j}$ in these locations and also in the single location $E_{k r}$ Other off-main diagonal blocks are 0 . If we reverse the factorization we do not change the main diagonal blocks, or any row above row $k$, and row $k$ is increased or stays the same. To the block $A_{k s}$ we have added

$$
E_{k r} A_{r s}=A_{k k}^{-1} A_{k r} A_{r s}
$$

Therefore by continuing this process we may assume

$$
A_{k s} \geq A_{k k}^{-1} A_{k r} A_{r s}
$$

for $s \leq r<k$. We may go down the rows until this holds for every block in the matrix. In particular,

$$
A_{k s}=A_{k k}^{-1} A_{k s} A_{s s} \quad\left(E_{i j}=\left(A_{i i}\right)^{-1} A_{i j}\right),
$$

by counting numbers of ones. These two equations, with main diagonal entries being permutations imply periodicity. In particular $A^{2}=P A$ where $P$ is the permutation matrix whose main diagonal blocks are the $A_{k k}$. So $A^{m}=P^{m-1} A$. This completes the proof.

\section{Distinguishing irreflexive primitives}

We begin with the following definition.

Definition. For a square Boolean matrix $A$ we say that $A$ is expandable to $C$ if by repeatedly splitting columns of $A$ and duplicating rows we can obtain $C$. If at each stage after the columns are split but before rows are duplicated the columns of $A$ contain at most one 1 we call the expansion a point expansion (p.e.). If $A \leq B$ and on every irreducible component $B_{i}$ of $B$ and for all $m, \operatorname{Tr}\left(A_{i}^{m}\right)=\operatorname{Tr}\left(B_{i}^{m}\right)$ where $A_{i}$ is the submatrix of $A$ in the same location as $B_{i}$ we say that we have a trace equivalence (t.e.).

Remark. Every expansion is a strong shift equivalence. 
In this section we go over some possible means of distinguishing Boolean matrices that are shift equivalent.

LEMMA 6.1. Suppose $E \leq F$ is a t.e. and $F_{1}$ is an expansion of $F$ and $E_{1}$ a corresponding p.e. of $E$, with $E_{1} \leq F_{1}$. Then $E_{1} \leq F_{1}$ is a t.e.

Proof. Indecomposable blocks of $F_{1}$ correspond to those of $F$. So we may restrict attention to an indecomposable block, and assume $F, F_{1}$ are indecomposable. But since $E, E_{1}$ are s.s.e. if $\operatorname{Tr}\left(E^{m}\right)=1$, then $\operatorname{Tr}\left(E_{1}^{m}\right)=1$.

LeMMA 6.2. Suppose $E \leq F$ and $F_{1}$ is an expansion of $F$. Then there exists $F_{2}$ obtained by replicating rows and columns of $F$ and $E_{2} \leq F_{2}$ such that $E_{2}$ is a p.e. of $E$.

Proof. At each stage of the expansion of $F$ to $F_{1}$, replicate the rows and columns of the expanded matrix enough times so that we can put the 1 entries in a column of $E$ in distinct columns of the expanded matrix from $F$. If columns are left over, take 0 columns in the expansion of $E$.

THEOREM 6.3. Let $A, B$ be two square Boolean matrices. If $A$ is strong shift equivalent to $B$ then there exists an iterated p.e. $C$ of $A$ such that for some conjugated block form $D$ of $B, C \leq D$ and this is a t.e.

Proof. We first show this relationship is transitive. Suppose $C$ is an iterated p.e. of $A, D$ is a conjugate block form of $B, C \leq D, E$ is an iterated p.e. of $B, F$ is a conjugated block form of $G, E \leq F$. We take $E_{1}$ to be a conjugated block form of $E$ which is an expansion of $D$ corresponding to the expansion $E$ of $B$. Then we can take the corresponding conjugated block form $F_{1}$ of $F$, and $E_{1} \leq F_{1}$ and $F_{1}$ is a conjugated block form of $G$.

We replicate all rows and columns of $E_{1}, F_{1}$ and call these $E_{2}, F_{2}$, where there exists a p.e. $E_{3}$ of $D$ such that $E_{3} \leq E_{2}$. Now we take a p.e. $C_{3}$ of $C$ corresponding to $E_{3}$ of $D$. Then $C_{3}$ is a p.e. of $A$ and $C_{3} \leq F_{2}$.

Now we show the inequalities are t.e. We have in turn that $E \leq F$ is a t.e., $E_{1} \leq F_{1}$ is, being a block form of it. So is $E_{2} \leq F_{2}$. And since $E_{3} \leq E_{2}$ is, $E_{3} \leq E_{2} \leq F_{2}$ is. And since $C \leq D$ is, $C_{3} \leq E_{3}$ is.

It remains to show that one step s.s.e.'s have this form. For $S R=A, R S=B$, factor $R$ as in proposition 3.2. So we may as before deal with two cases (i) a column of $A$ is split in two and the rows duplicated, (ii) for two column rows of $A$ we add the rows. If we take a block form in (ii) we may assume the dimension is unchanged, so that $A \leq B$ gives the required form.

Assume (i). Let $A_{*_{j}}$ be split into $v, w$. By taking a larger block form we may assume we have $k$ copies of each column in $B$ having $k$ ones. Now we take a p.e. $C$ of $A$ in which we put the $k$ th one in a column corresponding to the $k$ th copy of that column in $B$. If columns are left over (for $v, w$ ) then put zero columns in $A$. We do this first for the columns and then duplicate all the rows correspondingly. Then the p.e. $C$ of $A$ satisfies $C \leq B$.

By lemma 6.1 we have a t.e. This proves the theorem.

COROllary 6.4. Any matrix over $\mathbb{Z}^{+}$representing $A$ is strong shift equivalent over $\mathbb{Z}^{+}$to a matrix less than or equal to a block form of $B$. 
Proof. Any p.e. can be done over $\mathbb{Z}^{+}$.

Definition. A homomorphism from a graph with vertex and edge set $\left(V_{1}, E_{1}\right)$ to $\left(V_{2}, E_{2}\right)$ consists of a map $f\left(V_{1}\right) \rightarrow V_{2}$ such that if $(v, w) \in E_{1}$ then $(f(v), f(w)) \in E_{2}$. Remark. In many cases, no subset of the vertices of $B$ can yield a t.e.

Theorem 6.5. Let $A, B$ be two Boolean matrices. Let $A$ be s.s.e. to B. Let $R_{0}=\mathbb{R}^{+} \cap S$ where $S \subset \mathbb{R}$ is a subring containing 1 and some $x \in(0,1)$. Let $C$ be a matrix over $R_{0}$ whose Boolean form is $A$. Then there exists a matrix $D$ s.s.e. to $C$ over $R_{0}$ and having a conjugated Boolean block form of $B$ in which each block is entirely zero or entirely non-zero.

Proof. Every s.s.e. can be factored in terms of transformations and transpose transformations. It suffices to duplicate the effect of these constructions: (1) for two equal columns add the rows and delete the column, (2) split a column in two and add the rows, since transformations can be factored in terms of permutations, (3) if a row or a column is zero delete the row and the column. For (1) let columns $v, w$ have the same Boolean form. For sufficiently small $\delta$, the vectors $v-\delta w, w-\delta v$ have the same Boolean form. Take $\delta \in R_{0}$ with this property. Then we can solve

$$
\left[\begin{array}{ll}
u & z
\end{array}\right]\left[\begin{array}{cc}
1+\delta^{2} & \delta \\
\delta & 1
\end{array}\right]=\left[\begin{array}{ll}
v & w
\end{array}\right],
$$

where $u, z$ have the same Boolean image as $v, w$. Then replace $v, w$ by $u, z$ and apply

$$
\left[\begin{array}{cc}
1+\delta^{2} & \delta \\
\delta & 1
\end{array}\right]
$$

to the rows. For (2), split the column for (non-zero entries use $x, 1-x$ ) and duplicate the rows. Step (3) carries over immediately.

Corollary 6.6. Let $A$ be strong shift equivalent to $B$ over $\mathfrak{B}$. Then there exist $C, D$ over $\mathbb{Z}^{+}$such that $C$ has Boolean image $A, D$ has Boolean image a Boolean block form of $B$ where all blocks are entirely zero or entirely non-zero such that $C$ is strong shift equivalent to $D$ over $\mathbb{Z}^{+}$.

Proof. Take $R_{0}=\mathbb{Q}^{+}$, take multiples of $C, D$.

Proposition 6.7. Let $A, B$ be Boolean matrices. If $A$ is strong shift equivalent to $B$ then there exists a graph homomorphism from an iterated directed edge graph $E^{n}(A)$ to $B$ such that the inclusion of the image of $A$ in $B$ preserves the trace of every power of each irreducible component.

Proof. For matrices over $\mathbb{Z}^{+}$, this is a restatement of the Curtis-Hedlund-Lyndon theorem [3]. But by corollary 6.2, if $A$ is s.s.e. over $B$ to $B$ then we can get a strong shift equivalence over $\mathbb{Z}^{+}$if we replace $B$ by a block form $B_{1}$. But there exists an epimorphism from the graph of $B_{1}$ to that of $B$. Composing this with that from the graph of $A$ to the graph of $B$ gives the required mapping.

This result also follows directly from theorem 6.3.

Other invariants which do not seem to provide additional information are the trace of Boolean tensor product $A \otimes \cdots \otimes A$ and symmetric tensor powers. (S.s.e. 
is preserved by all homomorphisms) and s.s.e. types of various Boolean modules.

Definition. A Boolean module over a set $S$ is a subspace $W$ of a Boolean vector space and a transformation $f: W \rightarrow W$ such that $f(0)=0, f(x+y)=f(x)+f(y)$ for all $x, y \in W$ and that whenever two polynomials in $S$ are equal the two polynomials in $f$ are equal. Two modules $M_{1}, M_{2}$ are strong shift transforms (s.s.t.) if and only if there exist $f: M_{1} \rightarrow M_{2}$ and $g: M_{2} \rightarrow M_{1}$ such that for all $z \in M_{1}, y \in M_{2} ; f(g(y))=x y$, $g(f(z))=x z$. Modules linked by a sequence of s.s.t. are strong shift equivalent.

This concept turns out to be equivalent to shift (not strong shift) equivalence. In the $\mathbb{Z}$ case modules can yield shift equivalence invariants of interest.

THEOREM 6.8. Let $F_{n}$ denote the n-square Boolean matrix which is the complement of the identity, $f_{i j}=0$ if and only if $i=j$. For all $n>3$, the matrices $F_{n}$ are strong shift equivalent.

Proof. For $n>4$, we have $F_{n}$ is strong shift equivalent to $F_{k}$ if $n<k \leq\left(\begin{array}{l}n \\ 2\end{array}\right)$. The proof is as follows. Let $F_{k}$ be indexed on subsets $X, Y$ of $\{1,2, \ldots, n\}$ having exactly 2 elements. Let $S$ be the $k \times n$ matrix such that $S_{X_{j}}=1$ if and only if $j \notin X$. Let $R$ be the $n \times k$ complement of the transpose of $S$. Then $S R$ has $(X, Y)$-entry 1 if and only if for some $j \notin X, j \in Y$. This happens if and only if $X \neq Y$. So $S R=F_{k}$. And $R S$ has $(i, j)$-entry 1 if and only if for some set $X$ in the family, $i \in X$ and $j \notin X$. This is false if $i=j$. We can choose the family of sets to include all subsets $\{i, i+1\}$ modulo $n$. Then the sets containing $i$ are $\{i, i+1\}$ and $\{i, i-1\}$ and $j$ will not be in both. So $F_{n}$ is s.s.e. to $F_{4}$ because for $n \geq 4,\left(\begin{array}{c}n \\ 2\end{array}\right)>n$.

Example. Suppose we want to 4-colour the directed edge graph of the complete graph $K_{6}$. Let $X_{1}, \ldots, X_{6}$ be the 6 subsets $\{1,2\},\{1,3\},\{1,4\},\{2,3\},\{2,4\},\{3,4\}$. Colour an edge from $v_{i}$ to $v_{j}$ any colour $j$ such that $j \in X_{i}, j \notin X_{j}$. Then any edge from $v_{j}$ to $v_{k}$ will have a different colour.

Definition. For a graph $G=(E, V)$, let $F(G)$ have vertices all pairs $\mathscr{Y}, \mathscr{T} \subset V$ such that for all $x \in \mathscr{F}, y \in \mathscr{T} ;(x, y) \in E$ such that $\mathscr{S}$ is maximal with respect to given $\mathscr{T}$ and $\mathscr{T}$ is maximal with respect to given $\mathscr{T}$. The edges of $F(G)$ are all sequences $\left(\mathscr{S}_{1}, \mathscr{T}_{1}\right),\left(\mathscr{S}_{2}, \mathscr{T}_{2}\right)$ such that $\mathscr{S}_{1} \cap \mathscr{T}_{2} \neq \varnothing$. Let $F_{1}(G)$ denote this construction where maximality is omitted.

THEOREM 6.9. There exists a homomorphism $E(H) \rightarrow G$ for any directed graph $H$ if and only if there exists a homomorphism $H \rightarrow F(G)$.

Proof. Given $f: H \rightarrow F(G)\left(H \rightarrow F_{1}(G)\right)$ let $e$ be any edge $r \rightarrow s$ where $f(r)=\mathscr{S}_{1}, \mathscr{T}_{1}$ and $f(s)=\mathscr{S}_{2}, \mathscr{T}_{2}$. Then let $g(e)$ be any vertex in $\mathscr{T}_{1} \cap \mathscr{S}_{2}$. Then if $e_{1} \rightarrow e$ in $E(H)$, $g\left(e_{1}\right) \in \mathscr{S}_{1}$ so $\left(g\left(e_{1}\right), g(e)\right) \in \mathscr{S}_{1} \times \mathscr{T}_{1} \subset E$.

Conversely, let $g$ be a homomorphism from $E(H)$ to $G$. For a vertex $x_{1} \in H$, let $\mathscr{S}_{0}, \mathscr{T}_{0}$ be the edges of $H$ leading into and out of $x_{1}$. Let $\mathscr{S}_{1}=g\left(\mathscr{F}_{0}\right)$, $\mathscr{T}_{1}=g\left(\mathscr{T}_{0}\right)$. Then every vertex of $g\left(\mathscr{S}_{0}\right)$ joins every vertex of $g\left(\mathscr{T}_{0}\right)$. Choose a maximal family $g\left(\mathscr{T}_{0}\right) \subset \mathscr{T}, g\left(\mathscr{T}_{0}\right) \subset \mathscr{T}$. Let $f\left(x_{1}\right)=\mathscr{S}, \mathscr{T}$. If $\left(x_{1}, y\right) \in E$ where $f(y)=\mathscr{S}_{2}, \mathscr{T}_{2}$ then $\left(x_{1}, y\right) \in \mathscr{T}_{0}$ so then $g\left(x_{1}, y\right) \in \mathscr{T}$ and $g\left(x_{1}, y\right) \in \mathscr{S}_{2}$ by definition so $\mathscr{T} \cap \mathscr{S}_{2} \neq \varnothing$. 
THEOREM 6.10. For any non-zero matrix $A$ with trace zero, there exists a continuous map commuting with $\mathbb{S}$ from the subshift given by $A$ to the shift given by

$$
B=\left[\begin{array}{lll}
0 & 1 & 1 \\
0 & 0 & 1 \\
1 & 0 & 0
\end{array}\right] .
$$

There also exists a homomorphism $E^{n}(G(A)) \rightarrow G(B)$.

Proof. The second statement implies the first. Theorem 6.8 implies the existence of a homomorphism $E^{n}\left(K_{n}\right)$ to $E\left(K_{4}\right)$ and therefore $E^{n}(G(A))$ to $K_{4}$ where $K_{m}$ denotes the $m$ point complete graph.

Next we show that there is a map $E^{3}\left(K_{4}\right) \rightarrow K_{3}$. We will show $K_{4} \subset F_{1}^{3}\left(K_{3}\right)$. The function $F_{1}$ is monotone on graphs under inclusion. In $F\left(K_{3}\right)$, we have the subset of pairs $\mathscr{S}, \mathscr{T} ; a_{1}=(12,3), a_{2}=(13,2), a_{3}=(23,1)$, and $a_{4}=(1,23)$. These give the graph where $a_{1}, a_{2}, a_{3}$ and $K_{3}$ and $a_{4} \rightarrow a_{1}, a_{2}, a_{3}$ and $a_{3} \rightarrow a_{1}$.

In $F_{1}^{2}\left(K_{3}\right)$, we have the subset of pairs $a_{5}=\left(a_{3}, a_{4} a_{1} a_{2}\right), a_{6}=\left(a_{4} a_{1}, a_{2} a_{3}\right), a_{7}=$ $\left(a_{4} a_{2}, a_{1} a_{3}\right)$, and $a_{8}=\left(a_{4} a_{3}, a_{1} a_{2}\right)$. Then $a_{6}, a_{7}, a_{8}$ are a $K_{3}, a_{5} \rightarrow a_{6}, a_{7}, a_{8}$, and $a_{6}$, $a_{7} \rightarrow a_{5}$.

In $F_{1}^{3}\left(K_{3}\right)$, we have a $K_{4}$ given by $a_{9}=\left(a_{6} a_{7}, a_{5} a_{8}\right), a_{10}=\left(a_{5} a_{6}, a_{7} a_{8}\right), a_{11}=\left(a_{5} a_{7}\right.$, $\left.a_{6} a_{8}\right)$, and $a_{12}=\left(a_{5} a_{8}, a_{6} a_{7}\right)$.

Finally, we show there is a map $E^{4}\left(K_{3}\right) \rightarrow C$ where $C$ is the graph of a matrix strong shift equivalent to $B$, using $B_{*_{2}} \leq B *_{3}$,

$$
\left[\begin{array}{lll}
0 & 1 & 1 \\
1 & 0 & 1 \\
1 & 0 & 0
\end{array}\right] .
$$

$F(C)$ has vertices $b_{1}, b_{2}, b_{3}, b_{4}$ given by $(12,3),(23,1),(1,23),(2,13)$. We have $b_{i} \leftrightarrow b_{i+1}, b_{3}, b_{4} \rightarrow b_{1}, b_{2} . F_{1}^{2}(C)$ has vertices, among others $b_{8}=\left(b_{3}, b_{4} b_{2} b_{1}\right), b_{7}=$ $\left(b_{4} b_{2}, b_{3} b_{1}\right), b_{6}=\left(b_{4} b_{3}, b_{2} b_{1}\right), b_{5}=\left(b_{4} b_{3} b_{1}, b_{2}\right)$. Then $b_{j} \rightarrow b_{i}$ if $j>i, b_{7} \leftrightarrow b_{i}$ if $i \neq 7$. $F_{1}^{3}(C)$ has vertices, among others $b_{12}=\left(b_{7}, b_{8} b_{6} b_{5}\right), b_{11}=\left(b_{8} b_{7}, b_{6} b_{5}\right), \quad b_{10}=$ $\left(b_{8} b_{6}, b_{7} b_{5}\right), b_{9}=\left(b_{8} b_{6} b_{5}, b_{7}\right)$. Then $b_{12} \leftrightarrow b_{10} \leftrightarrow b_{11} \leftrightarrow b_{9} \leftrightarrow b_{12}$ and $b_{12} \rightarrow b_{11}, b_{10} \rightarrow b_{9}$.

In $F_{1}^{4}(C)$, we have a $K_{3},\left(b_{12} b_{11}, b_{10} b_{9}\right),\left(b_{10} b_{9}, b_{12} b_{11}\right)$, and $\left(b_{12} b_{10}, b_{11} b_{9}\right)$.

\section{Conclusion}

S.s.e. of most Boolean matrices is decided by s.e. and traces of all powers of all irreducible components. Two matrices are Boolean strong shift equivalent if and only if some matrices over $\mathbb{Z}^{+}$having block forms of them as images are. The question of continuous mappings between subshifts commuting with $S$ can be decided from their Boolean images.

Dual edge graphs $F(G)$ give a possible method of solving the problems involved. A major question remains: for $A, B$ over $B$ primitive and $\operatorname{Tr}\left(A^{n}\right)=\operatorname{Tr}\left(B^{n}\right)$ all $n$, are $\boldsymbol{A}, \boldsymbol{B}$ strong shift equivalent? If the answer is false it could give new s.s.e. invariants. 
THEOREM 7.1. Two Boolean matrices of size at most $3 \times 3$ are strong shift equivalent if and only if they are shift equivalent and the traces of all powers of corresponding main diagonal indecomposable blocks are equal.

Proof. This follows from theorem 5.4 except for primitives of trace zero (none exist for $2 \times 2$ ). There are these conjugacy classes of trace zero primitives

$$
\left[\begin{array}{lll}
0 & 1 & 1 \\
0 & 0 & 1 \\
1 & 0 & 0
\end{array}\right],\left[\begin{array}{lll}
0 & 1 & 1 \\
0 & 0 & 1 \\
1 & 1 & 0
\end{array}\right],\left[\begin{array}{lll}
0 & 1 & 1 \\
1 & 0 & 1 \\
1 & 1 & 0
\end{array}\right]
$$

The first two are strong shift equivalent by adding column 1 to column 2 . We show the last two are.

We take $9 \times 9$ matrices of $3 \times 3$ blocks and repeatedly factor a $3 \times 3$ matrix out of $h$ row (column) block on the left (right) and multiply in into the column (row) block on the right (left), after initial conjugation.

Let

$$
\begin{aligned}
& A=\left[\begin{array}{lll}
0 & 1 & 1 \\
0 & 0 & 1 \\
1 & 1 & 0
\end{array}\right], \quad B=\left[\begin{array}{lll}
0 & 1 & 1 \\
1 & 0 & 1 \\
1 & 1 & 0
\end{array}\right], \quad J=\left[\begin{array}{lll}
1 & 1 & 1 \\
1 & 1 & 1 \\
1 & 1 & 1
\end{array}\right], \quad X=\left[\begin{array}{lll}
1 & 1 & 1 \\
0 & 0 & 1 \\
1 & 1 & 0
\end{array}\right] \\
& Y=\left[\begin{array}{lll}
1 & 1 & 1 \\
0 & 1 & 0 \\
0 & 0 & 1
\end{array}\right], \quad U=\left[\begin{array}{lll}
1 & 1 & 0 \\
1 & 1 & 0 \\
0 & 0 & 1
\end{array}\right], \quad V=\left[\begin{array}{lll}
1 & 1 & 1 \\
1 & 1 & 0 \\
1 & 1 & 1
\end{array}\right], \quad W=\left[\begin{array}{lll}
0 & 1 & 1 \\
0 & 1 & 1 \\
1 & 1 & 0
\end{array}\right] \\
& K=\left[\begin{array}{lll}
1 & 1 & 0 \\
1 & 1 & 0 \\
1 & 1 & 1
\end{array}\right], \quad A^{2}=\left[\begin{array}{lll}
1 & 1 & 1 \\
1 & 1 & 0 \\
0 & 1 & 1
\end{array}\right], \quad B^{2}=J
\end{aligned}
$$

These are conjugate:

$$
\left[\begin{array}{lll}
0 & B & B \\
0 & 0 & B \\
B & B & 0
\end{array}\right], \quad\left[\begin{array}{ccc}
0 & A & A \\
A & 0 & A \\
A & A & 0
\end{array}\right]
$$

The first matrix is s.s.e. in turn to

$$
\begin{aligned}
& {\left[\begin{array}{ccc}
0 & B^{2} & B^{2} \\
0 & 0 & B \\
I & B & 0
\end{array}\right],\left[\begin{array}{lll}
0 & J & J \\
0 & 0 & B \\
I & B & 0
\end{array}\right],\left[\begin{array}{lll}
0 & J & J \\
0 & 0 & B \\
J & B & 0
\end{array}\right],\left[\begin{array}{ccc}
0 & J & J \\
0 & 0 & I \\
J & B^{2} & 0
\end{array}\right]} \\
& {\left[\begin{array}{lll}
0 & J & J \\
0 & 0 & I \\
J & J & 0
\end{array}\right],\left[\begin{array}{lll}
0 & J & J \\
0 & 0 & J \\
J & J & 0
\end{array}\right], A .}
\end{aligned}
$$

The second matrix is s.s.e. to (using $V=V K, X A=A^{2}, Y^{2}=Y, Y X=X, X W=A^{2}$, $U A=W, A X=V, A Y V=K Y=K V=J)$,

$$
\left[\begin{array}{ccc}
0 & A^{2} & A^{2} \\
I & 0 & A \\
I & A & 0
\end{array}\right],\left[\begin{array}{ccc}
0 & X W & X W \\
I & 0 & A \\
I & A & 0
\end{array}\right],\left[\begin{array}{ccc}
0 & W & W \\
X & 0 & A \\
X & A & 0
\end{array}\right],\left[\begin{array}{ccc}
0 & U A & W \\
X & 0 & A \\
X & A & 0
\end{array}\right]
$$




$$
\begin{aligned}
& {\left[\begin{array}{ccc}
0 & U & W \\
A X & 0 & A^{2} \\
X & I & 0
\end{array}\right],\left[\begin{array}{ccc}
0 & U & U A \\
A X & 0 & X A \\
X & I & 0
\end{array}\right],\left[\begin{array}{ccc}
0 & U & U \\
A X & 0 & X \\
A X & A & 0
\end{array}\right]} \\
& {\left[\begin{array}{lll}
0 & U & U \\
V & 0 & X \\
V & A & 0
\end{array}\right],\left[\begin{array}{lll}
0 & K & K \\
V & 0 & X \\
V & A & 0
\end{array}\right],\left[\begin{array}{ccc}
0 & K & K \\
Y V & 0 & Y X \\
V & A & 0
\end{array}\right],\left[\begin{array}{ccc}
0 & K Y & K \\
V & 0 & X \\
V & A Y & 0
\end{array}\right]} \\
& {\left[\begin{array}{ccc}
0 & J & K \\
V & 0 & X \\
V & A Y & 0
\end{array}\right],\left[\begin{array}{ccc}
0 & J & K \\
A Y V & 0 & A Y X \\
V & I & 0
\end{array}\right],\left[\begin{array}{ccc}
0 & J & K \\
J & 0 & V \\
V & I & 0
\end{array}\right]} \\
& {\left[\begin{array}{ccc}
0 & J & I \\
J & 0 & V \\
K V & K & 0
\end{array}\right],\left[\begin{array}{lll}
0 & J & I \\
J & 0 & V \\
J & K & 0
\end{array}\right],\left[\begin{array}{lll}
0 & J & J \\
J & 0 & V \\
J & K & 0
\end{array}\right]} \\
& {\left[\begin{array}{ccc}
0 & J & J \\
J & 0 & K V \\
J & I & 0
\end{array}\right],\left[\begin{array}{lll}
0 & J & J \\
J & 0 & J \\
J & I & 0
\end{array}\right],\left[\begin{array}{lll}
0 & J & J \\
J & 0 & J \\
J & J & 0
\end{array}\right]}
\end{aligned}
$$

Acknowledgments. The authors would like to thank Mike Boyle, Bruce Kitchens, Bob Williams, and an unknown referee for very constructive criticism and suggestions.

\section{REFERENCES}

[1] K. A. Baker. Strong shift equivalence of $2 \times 2$ matrices of non-negative integers. Ergod. Th. Dynam. Sys. 3 (1983), 541-558.

[2] M. Boyle. Private correspondence, 1984.

[3] G. A. Hedlund. Endomorphisms and automorphisms of the shift dynamical system. Math. Sys. Theory 3 (1969), 320-375.

[4] K. H. Kim. Boolean Matrix Theory and Applications. Marcel Dekker, 1982.

[5] K. H. Kim \& F. W. Roush. Some results on the decidability of shift equivalence. Jour. of Combinatorics, Information, and Systems Sciences 4 (1979), 123-146.

[6] K. H. Kim \& F. W. Roush. The algebraic structure of the semigroup of binary relations on a finite set. Glasgow Math. Jour. 22 (1981), 57-68.

[7] W. Parry \& R. F. Williams. Block coding and a zeta function for finite Markov chains. Proc. London Math. Soc. 35 (1977), 483-495.

[8] R. F. Williams. Classification of symbol spaces of finite type. Bull. Amer. Math. Soc. 77 (1971), 439-443.

[9] R. F. Williams. Classification of subshifts of finite type. Ann. Math. 98 (1973), 120-152. Errata, ibid. 99 (1974), 380-381. 